\title{
Modeling galvanostatic charge-discharge of nanoporous supercapacitors
}

\section{Liang Zeng}

Huazhong University of Science and Technology

\section{Taizheng Wu}

Huazhong University of Science and Technology

\section{Ting Ye}

Huazhong University of Science and Technology

\section{Tangming Mo}

Huazhong University of Science and Technology

\section{Rui Qiao}

Virginia Tech

\section{Guang Feng ( $\square$ gfeng@hust.edu.cn )}

Huazhong University of Science and Technology https://orcid.org/0000-0001-6659-9181

\section{Article}

Keywords: constant potential method (CPM), supercapacitors, molecular modeling

Posted Date: May 27th, 2021

DOl: https://doi.org/10.21203/rs.3.rs-488710/v1

License: (c) (i) This work is licensed under a Creative Commons Attribution 4.0 International License. Read Full License

Version of Record: A version of this preprint was published at Nature Computational Science on November 22nd, 2021. See the published version at https://doi.org/10.1038/s43588-021-00153-5. 


\section{Modeling galvanostatic charge-discharge of nanoporous}

\section{2 supercapacitors}

3 Liang Zeng ${ }^{1}$, Taizheng $\mathrm{Wu}^{1}$, Ting $\mathrm{Ye}^{1}$, Tangming Mo ${ }^{1}$, Rui Qiao ${ }^{2}$, and Guang Feng ${ }^{1, *}$

4

5 Molecular modeling can study the energy storage of supercapacitors at the atomistic

6 level and has become indispensable in this research. The constant potential method

7 (CPM) allows keeping the electric potential uniform on the electrode, which is essential

8 for a realistic description of the charge repartition and dynamics process in

9 supercapacitors. Prior CPM studies have been limited to the potentiostatic mode.

10 Though widely adopted in the experiment, the galvanostatic mode has been rarely

11 investigated in CPM simulations due to a lack of effective methods. In this work, we

12 developed a modeling approach to simulating the galvanostatic charge-discharge of

13 supercapacitors under constant potential (GCD-CPM). We show that, for nanoporous

14 electrodes, GCD-CPM can capture supercapacitor dynamics in excellent agreement

15 with experimental measurements and delineate the ion adsorption-desorption dynamics

16 underlying the hysteresis with molecular resolutions during charging and discharging.

17 Therefore, this GCD-CPM modeling could open up new avenues for exploring the rich

18 physics and electrochemistry of supercapacitor dynamics.

\footnotetext{
${ }^{1}$ State Key Laboratory of Coal Combustion, School of Energy and Power Engineering, Huazhong University of Science and Technology (HUST), Wuhan 430074, China. ${ }^{2}$ Department of Mechanical Engineering, Virginia Tech, Blacksburg, VA 24061, USA. E-mail: gfeng@ hust.edu.cn
} 
Supercapacitors have been attracting significant attention due to their high power density and superior cycle time ${ }^{1,2}$. Researchers frequently resort to molecular modeling to investigate the thermodynamics and dynamics of the energy storage in supercapacitors, particularly those with nanoporous electrodes, because molecular simulations can provide a precise microscopic picture of electric double layers (EDLs) and their formation in supercapacitors ${ }^{3-5}$. In the molecular modeling of supercapacitors, how to describe electrode polarization is a critical issue. The most straightforward way is to distribute charges uniformly on electrode atoms (i.e., constant charge method, $\mathrm{CCM})^{4-7}$. In contrast, the constant potential method (CPM), a computationally expensive but realistic approach, maintains electrode atoms at constant potential, adjusting the electrode charges self-consistently based on the electrode potential and ionic environment ${ }^{8-15}$. To explore the equilibrium performance of supercapacitors, CCM may be feasible for systems with open electrodes (e.g., those with planar $^{16}$, cylindrical $^{7}$, spherical $^{17}$ surfaces), but not for porous electrodes ${ }^{11-13,18,19}$. To model the charging and discharging process of supercapacitors, CPM is desired because CCM will produce incorrect charging dynamics and heat generation ${ }^{19,20}$.

In studying charging and discharging supercapacitors, prior CPM simulations often adopted the potential control mode: step-like, ${ }^{8-15}$ linear, $^{21}$ or climbing-type ${ }^{22,23}$ potential differences were applied between the positive and negative electrodes. These molecular simulations help to understand the fundamentals of charging and discharging supercapacitors. However, they cannot offer insights into the charging kinetics in the galvanostatic mode that has been widely used in practical applications ${ }^{24,25}$ and fundamental electrochemical studies (e.g., galvanostatic charge-discharge, GCD $)^{26-28}$. Therefore, modeling the charging and discharging of supercapacitors by regulating electric current and keeping electrode equipotential is highly desirable. Nevertheless, very few such studies are reported due to a lack of effective simulation methods. An approach combining finite electric displacement and constant potential technique was developed very recently but has been demonstrated so far for open electrode systems ${ }^{29}$. As such, existing GCD simulations were based on CCM, in which charges on electrode atoms were varied linearly and uniformly (in the following, this method is termed GCD-CCM) ${ }^{30,31}$. 
Herein taking inspiration from previous CPM studies ${ }^{8,9,13,15}$, we develop a method to model the galvanostatic charge-discharge of supercapacitors under constant potential, rigorously making electrode equipotential at each time step (named GCD-CPM, see Methods). Both GCD-CPM and GCD-CCM have been applied to two typical molecular dynamics (MD) systems of supercapacitors with open electrodes (Fig. 1a) and nanoporous electrodes (Fig. 1b). The ionic liquid ethyl-3-methyl-imidazolium tetrafluoroborate ([EMIM] $\left.\left[\mathrm{BF}_{4}\right]\right)$ is used in both systems (Fig. 1c). After equilibrating the simulation under the potential of zero surface charge, we apply a square-wave current with an amplitude of $I_{0}$ and a period of $P$ (Fig. 1d) (see Methods). We then analyze the time evolution of the electrode potential and EDL formation in both systems and demonstrate the effectiveness of GCD-CPM via experimental validation.

a

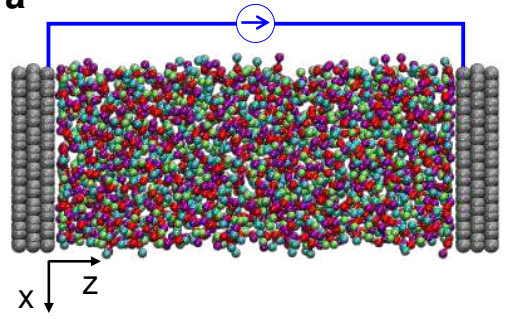

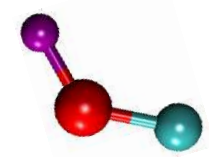

$[\mathrm{EMIM}]^{+}$

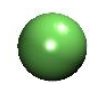

$\left[\mathrm{BF}_{4}\right]^{-}$ d

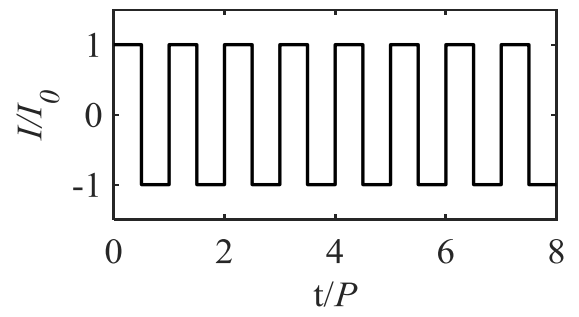

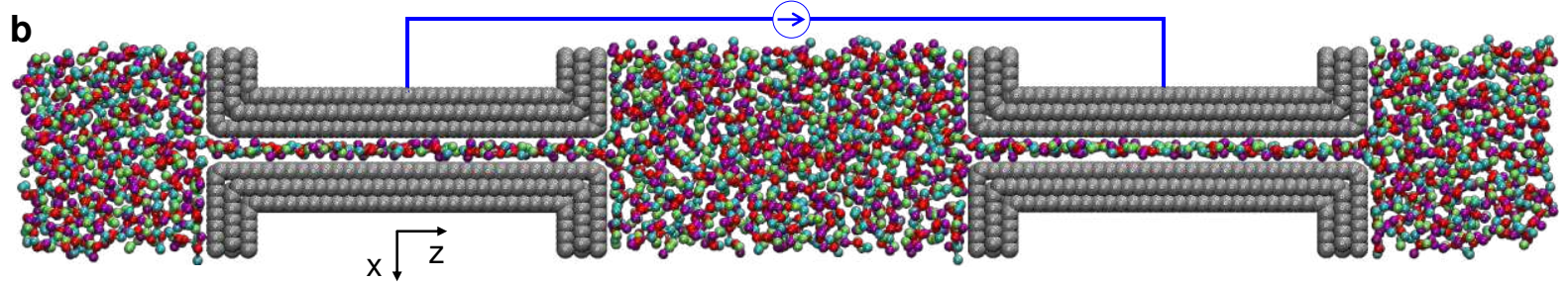

Fig. 1 | Molecular simulations of the galvanostatic charge-discharge of supercapacitors. a-b,

60 Snapshots of supercapacitors featuring open electrodes (a) and nanoporous electrodes (b). c,

61 Coarse-grained models of the ionic liquid [EMIM] $\left[\mathrm{BF}_{4}\right]$ used in supercapacitors. d, The applied 62 electric current of galvanostatic charge-discharge.

\section{Results}

\section{GCD-CPM and GCD-CCM produces very similar results for open electrode systems}

We first study the open electrode system. Like the GCD test in experiments, MD simulation systems are charged and discharged with a square-wave current for 30 cycles. Figure 2 shows modeling-obtained GCD of the stable cycle with a period of 100 ps (see Supplementary Part 1 for details). In molecular modeling with GCD-CPM, the charges distributed on electrode atoms are not homogenous at any instant (Fig. 2a), owing to the thermal motion of electrolyte 
molecules $^{5,32}$, which is in line with previous works using CPM with step-like potentials ${ }^{4,5,32}$.

Such heterogeneity can be quantified by the probability distribution of charges on electrode atoms $^{5,32}$. Corresponding to the applied current (Fig. 1d), such probability distributions are analyzed from GCD-CPM simulations for each time step during the charging-discharging process (see Supplementary Part 2 for detailed probability distribution calculation). They are in a volcano-like shape, and their width (or variance) changes a little with time (Fig. $2 \mathrm{~b}$ for the positive electrode and Supplementary Fig. 2a for the negative electrode). On the electrolyte side, cations and anions, driven by the electrode polarization, form dynamic EDLs, which are delineated by the ion number density as a function of time and distance from the electrode surface (Fig. 2c and Supplementary Fig. 3-4). One can see that EDLs have typical layering structures, consistent with previous observations ${ }^{4,33-35}$. During the charging process $(0 \sim 50 \mathrm{ps})$, the first cation layer decreases with time and is slightly away from the electrode surface, while this trend is reversed during discharging $(50 \sim 100 \mathrm{ps})$.
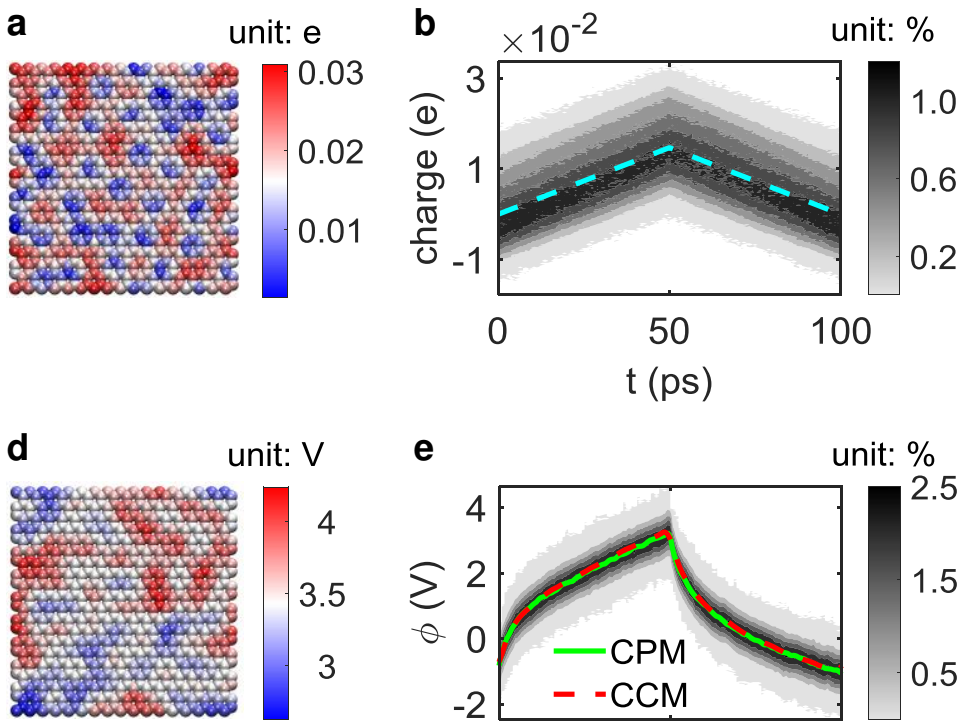
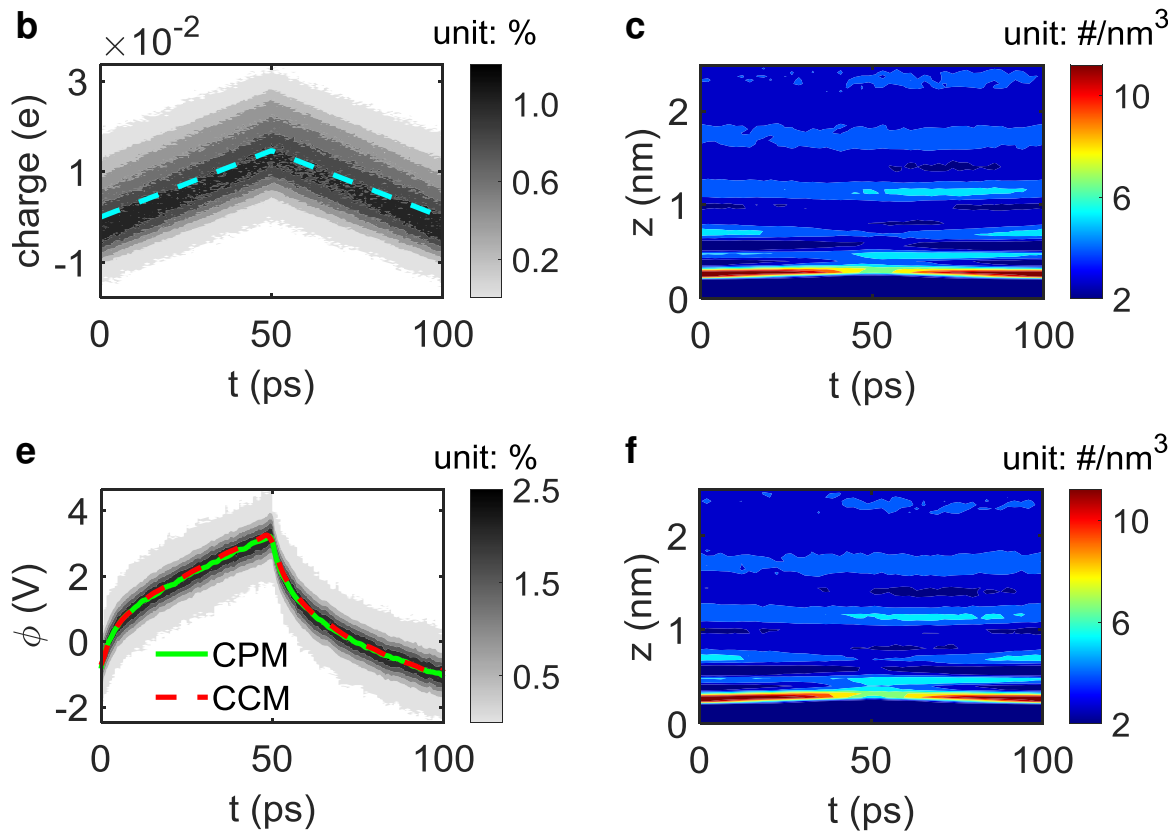

83

Fig. 2 | Galvanostatic charge-discharge of open electrode systems. a-c, GCD-CPM modeling: A snapshot of the positive electrode at $50 \mathrm{ps,}$ with atoms colored by their instantaneous charges (a); Gray contour presents probability distributions of electrode charges varying with time, and the dashed line is the average (b); Evolution of ion number density with time and distance from the electrode (c). d-f, GCD-CCM modeling: A snapshot of the positive electrode at $50 \mathrm{ps,} \mathrm{with} \mathrm{atoms} \mathrm{colored} \mathrm{by} \mathrm{their}$ instantaneous potential (d); Gray contour indicates probability distributions of electrode potentials, and the red dashed line is the average (e); Evolution of cation number density (f). The solid green line in (e) is the electrode potential obtained by GCD-CPM. 
The galvanostatic charge-discharge of the open electrode system is also explored using GCD-CCM. Instead of calculating charges distributed on electrode atoms at each time step in

94 GCD-CPM modeling, the charge on each electrode atom is preset with the same value in

95 GCD-CCM modeling. The electrode potential is computed accordingly, showing a distinctly heterogeneous distribution (Fig. 2d). However, the averaged potential on the electrode at each time closely matches that by GCD-CPM (Fig. 2e and Supplementary Fig. 2b). Evolution of cation density at the positive electrode computed by GCD-CCM is also found to be nearly the same as that by GCD-CPM (Fig. 2f vs. 2c); comparison of more dynamic EDL structures reaches the same agreement (Supplementary Fig. 3-4). With further checking the ion orientation, the angular distributions of cations adsorbed on the negative electrode obtained by these two methods are almost the same (Supplementary Fig. 5). For additional electric currents in different amplitudes and periods, the results obtained by these two methods again come to the same conclusion (Extended Data Fig. 1).

\section{GCD-CPM is indispensable for nanoporous electrode systems}

106 We then investigate the galvanostatic charge-discharge of nanoporous electrode systems. In 107 GCD-CPM modeling, the probability distributions of electrode charges are in more 108 substantial heterogeneity and vary with time more distinctively (Fig. 3a and Supplementary 109 Fig. 6a), compared with open electrode systems (Fig. 2b and Supplementary Fig. 2a). It could 110 be ascribed to the non-uniform distribution of electrode charges along the pore axis

111 (Extended Data Fig. 2a). In particular, the variation of surface charges at the pore entrance is 112 more extensive than those in the central portion of the pore. Driven by the electrode 113 polarization, the in-pore charge density $\rho_{e}$, stemming from the electrolyte, exhibits a similar 114 trend with the varied electrode charges (Fig. 3b vs. Extended Data Fig. 2a).

115 The potential distributions on nanoporous electrodes were examined via GCD-CCM 116 (Fig. 3c and Supplementary Fig. 6b), compared to GCD-CPM. It can be found that the 117 potential is more heterogeneous than that on the open electrode, and its average differs from 118 GCD-CPM. Remarkably, there is a parabolic-like distribution of the electrode potential from 
119 the entrance to the central portion of a pore (Extended Data Fig. 2b), which conflicts with the

120 fact that the electrode is equipotential. For GCD-CCM, the in-pore charges, driven by the 121 uniformly distributed electrode charges, are less heterogeneous than those obtained using 122 GCD-CPM (Fig. 3d vs. 3b).

a

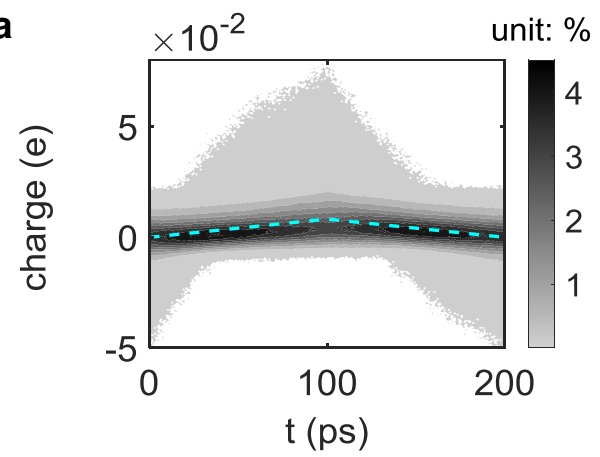

C

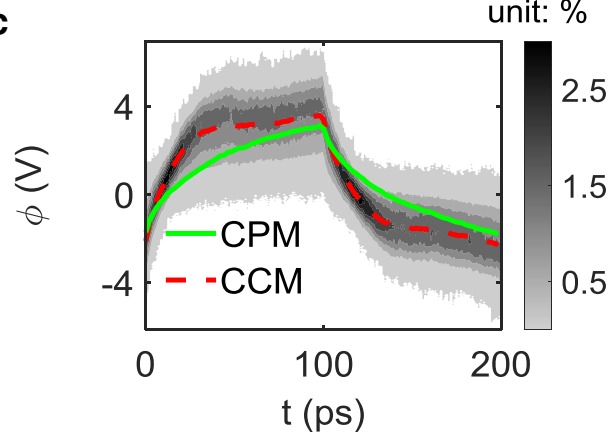

$\mathbf{e}$

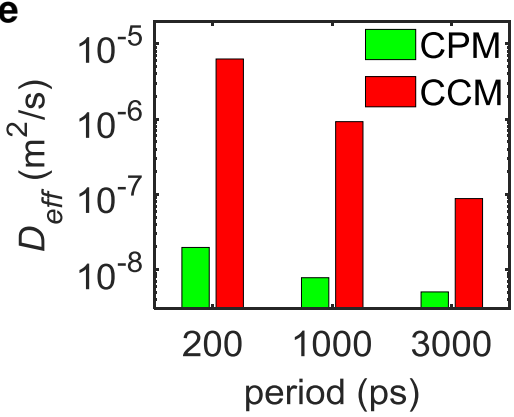

b

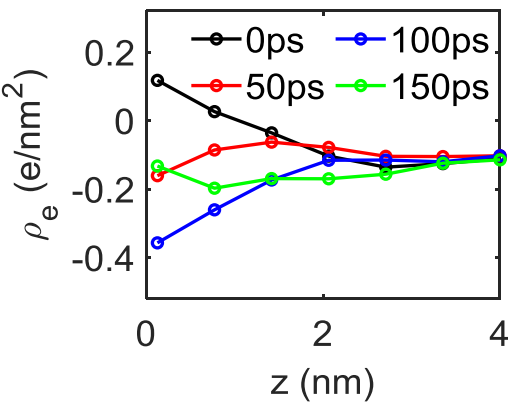

d

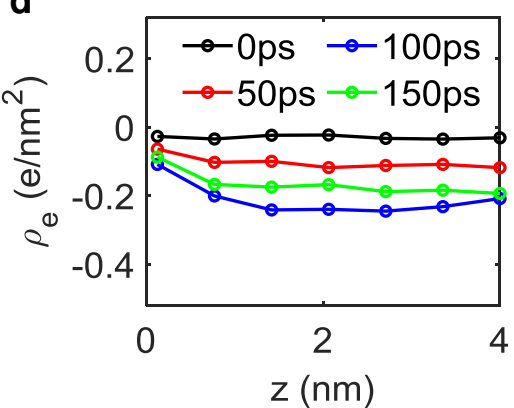

f

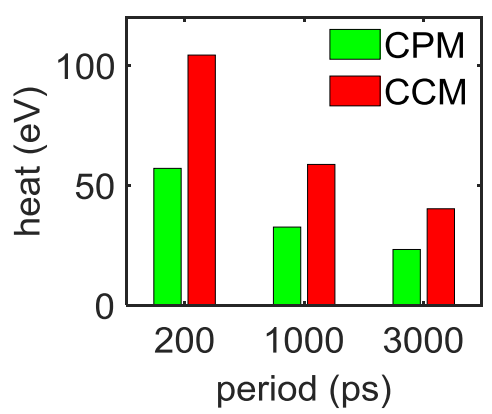

Fig. 3 | GCD-CPM and GCD-CCM modeling of galvanostatic charge-discharge in nanoporous electrode systems. a, Gray contour indicates probability distributions of atom charges on the positive electrode, obtained from GCD-CPM simulations; the dashed line is the average of such charges. $\mathbf{b}$, Evolution of in-pore charge density from electrolyte along the pore $(\mathrm{z}=0$ is the pore entrance), obtained from GCD-CPM simulations. c, Gray contour indicates probability distributions of potentials on the positive electrode, obtained from GCD-CCM simulations; the red dashed line is the average of potentials; the solid green line is the potential obtained from GCD-CPM simulations. d, Evolution of in-pore charge density from the electrolyte, obtained from GCD-CCM simulations. e, Effective diffusivity of in-pore electrolyte under the positive polarization. f, Heat generation during a galvanostatic charging-discharging cycle. The pore width is $0.67 \mathrm{~nm}$. 

the charging dynamics and power density of porous supercapacitors ${ }^{1,3,12}$. The in-pore ion transport during the charging-discharging process can be evaluated by the effective diffusivity, which is described as ${ }^{12}$

$$
\frac{\partial \rho_{e}}{\partial t}=D_{e f f} \frac{\partial \rho_{e}^{2}}{\partial^{2} z}
$$

139 Using Eq. 1 and the evolution of in-pore charge density (Fig. 3 and Supplementary Fig. 7),

140 the effective diffusivity $D_{\text {eff }}$ of electrolyte in positive nanoporous electrodes is calculated 141 and shown in Fig. 3e (see Supplementary Part 5 for calculation details). Using GCD-CPM 142 modeling, the effective diffusivity in the pore of the positive electrode relative to that of bulk 143 electrolyte $D_{\text {eff }} / D_{\text {bulk }}$ is 52 ( $\left.P=200 \mathrm{ps}\right), 21(P=1000 \mathrm{ps})$, and 13 ( $\left.P=3000 \mathrm{ps}\right)$, close to the

144 prior work using CPM with step-like potentials ${ }^{12,36}$. For GCD-CCM modeling, $D_{e f f} / D_{b u l k}$ 145 is $16566(P=200 \mathrm{ps}), 2434(P=1000 \mathrm{ps})$, and $231(P=3000 \mathrm{ps})$, which is quite larger than that 146 from GCD-CPM. A similar trend is found in the negative nanoporous electrodes 147 (Supplementary Fig. 8-9).

148 The previous simulation reported that unphysically huge heat would be generated during 149 the charging process by CCM modeling with inputting step-like charges ${ }^{19}$. The heat generated 150 during a charging-discharging cycle of nanoporous supercapacitors is calculated as the energy 151 removed from the system by the thermal bath (detail of heat calculation in Methods). Figure 152 3f and Supplementary Fig. 10 disclose that the heat generated by GCD-CCM is much more massive than that by GCD-CPM. Therefore, GCD-CCM cannot be used for modeling nanoporous supercapacitor dynamics.

\section{Experimental validation of GCD-CPM modeling}

156 We now validate molecular modeling with GCD-CPM through experimental galvanostatic

157 measurements. The supercapacitor takes the same electrolyte as modeled in our simulation.

158 The nanoporous carbon electrode has the averaged pore size very close to that in the 159 molecular models (experimental details are in Supplementary Part 7). In the experiment, the 
160 GCD curve has an IR drop caused by electrode resistance (Supplementary Fig. 12) ${ }^{26,37,38 .}$

161 Because the electrode resistance is neglected in molecular modeling, the IR drop is removed 162 from experimental GCD curves, as described in Supplementary Part 7. Such treatment helps 163 compare the experiment and modeling but does not change the charging and discharging of 164 electrolyte in porous electrodes (Fig. 4a vs. Supplementary Fig. 12a).
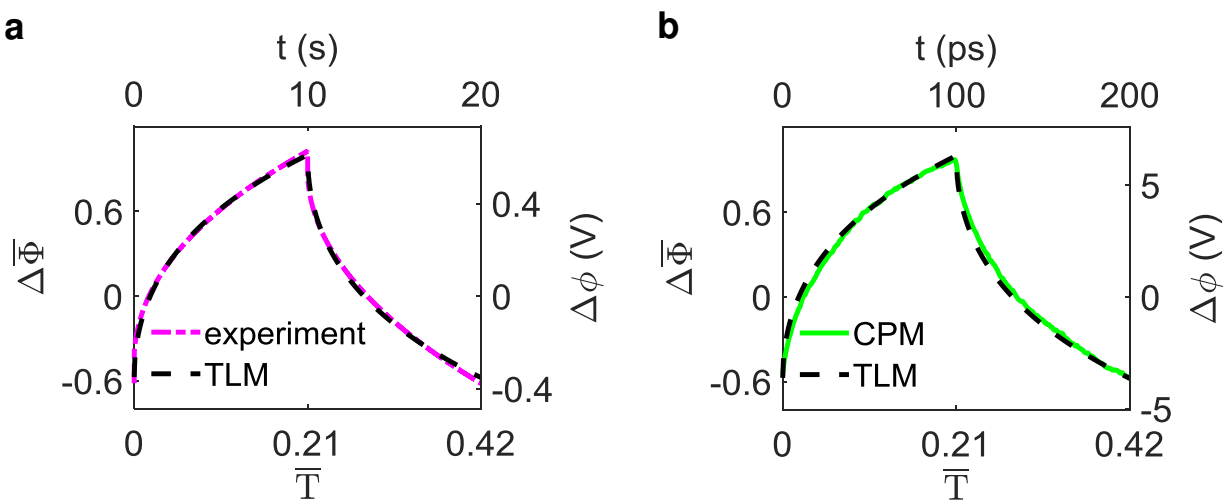

165

Fig. 4 | Comparison of GCD curves obtained from experiment and modeling. a, The GCD curve in the experiment with a period of $20 \mathrm{~s}$ and its fitting by the dimensionless TLM. $\mathbf{b}$, The GCD curve of GCD-CPM with a period of 200 ps and its fitting curve by the dimensionless TLM.

The charging dynamics of nanoporous electrodes can be illuminated by the transmission line model $(\mathrm{TLM})^{12,39-42}$. To bridge macroscale experiments and nanoscale modeling, we derive the dimensionless form of TLM for the charging and discharging process of nanoporous supercapacitors and find that the dimensionless GCD curve, described by the dimensionless potential $\Delta \bar{\Phi}$ varying with the dimensionless time $\overline{\mathrm{T}}$, is only determined by the dimensionless period $\alpha$ (defined as the ratio of the electric current period to the system charging time constant). Details of the dimensionless TLM can be found in Methods. As shown in Fig. 4a, the dimensionless TLM provides an excellent fitting of the experimental GCD data, with a dimensionless period of 0.42. It is then used to fit the GCD curve also with $\alpha=0.42$, obtained by GCD-CPM modeling of nanoporous systems (Fig. 4b), which presents a good agreement as well. Since GCD curves in the experiment and modeling have the same dimensionless period, their dimensionless TLM-fitted curves are the same, even though the actual periods in the experiment and modeling are $20 \mathrm{~s}$ and $200 \mathrm{ps}$, respectively. With regulating the electric current period, GCD-CPM modeling is well consistent with the 
183 experiment (Supplementary Fig. 13), bridged by the dimensionless TLM. However, the

184 fitting reveals an apparent discrepancy between the experiment and GCD-CCM modeling 185 (Supplementary Fig. 14). As a result, only GCD-CPM could predict the correct dynamic 186 process of charging and discharging nanoporous electrodes.

\section{Hysteresis in the charging-discharging process}

188 Figure 5a shows a hysteresis of ion adsorption-desorption during the charging-discharging

189 process of nanoporous supercapacitors, which can be evidenced by the time evolution of the

190 in-pore electrolyte density. Specifically, in the positive porous electrode, counterions (i.e., 191 anions) are attracted into pores during charging and rejected out of pores during discharging,

192 while a reversed trend exists for co-ions (i.e., cations). The desorption of counterions during

193 discharging does not backtrack to the adsorption during charging, and the change of co-ions

194 in the desorption process is also different from that in the adsorption process. These findings

195 illustrate that there is a hysteresis of ion response during the charging-discharging process.

196 Similar hysteresis loop has been experimentally observed in porous supercapacitors probed

197 by the electrochemical quartz crystal microbalance (note that the areal densities shown here

198 are based on the specific surface area of the porous electrode rather than the area of 199 quartz-crystal surface used in experiments) $)^{43-46}$.
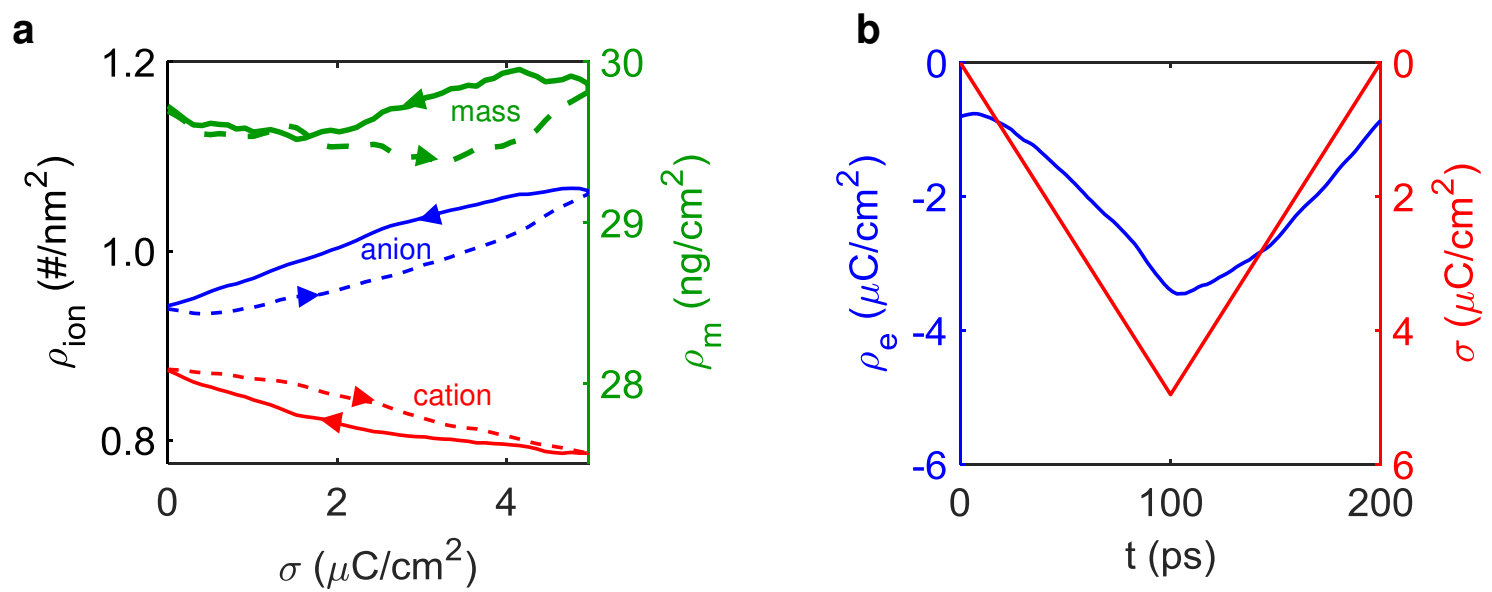

200

201

202

203

204

205

Fig. 5 | Hysteresis in nanoporous electrode systems. a, Green line is the mass density of in-pore electrolyte. Red and blue lines are the number densities of in-pore cations and anions, respectively. Solid (dashed) line is the discharging (charging) process. b, Blue line represents the charge density coming from the in-pore electrolyte, and red line is the charge density on the electrode. Results are for the positive electrode. 
The hysteresis of ion adsorption-desorption can be understood by comparing the charges

207 from the in-pore electrolyte and on the electrode (Fig. 5b). The charge change from the in-pore electrolyte exhibits a trend (increase or decrease with time) essentially the same as that on the electrode, but the change rate in the electrolyte side is slower than that in the

210 electrode side. It reflects that ion transport in the electrolyte, especially under 211 nanoconfinement, is sluggish compared to the charge transport through the electrode. As 212 shown in Fig. 5b, at the beginning of a stable cycle at the positive electrode $(0 \mathrm{ps})$, the total 213 charge on the electrode is zero, while that from the electrolyte is still negative; at the time 214 when the charging and discharging switch (100 ps), the charge from the in-pore electrolyte, in magnitude, is less than that from the electrode. Moreover, the charge change from the in-pore electrolyte in the initial stage is slower than in the rest, since both cations and anions change at a lower rate in the initial stage of the charging and discharging process (Fig. 5a). The hysteresis in the negative electrode exhibits the same trend (Supplementary Fig. 15).

There is also a hysteresis of the ion adsorption-desorption during charging and discharging in the open electrode system (Supplementary Fig. 16), though weaker than the nanoporous electrode system. Such hysteresis originates from the fact that the electrolyte ions do not respond to the electrode polarization as timely as the electrode charges do (Supplementary Fig. 17a). This hysteresis could account for the negative potentials occurring at the initial stage of the charging and the end of the discharging at the positive electrode (Fig. 2e and Extended Data Fig. 1). The details of the hysteresis and its contribution to the negative potential can be found in Supplementary Part 8 with Supplementary Fig. 16-17. Similarly, the negative value of potentials on the positive electrode of nanoporous supercapacitors can also be understood by the hysteresis of ion adsorption-desorption resulting in the negative charge from the electrolyte at the beginning of charging or the end of discharging (Fig. 5b).

\section{Discussion}

231 We developed a molecular simulation method to model the galvanostatic charge-discharge of 232 supercapacitors by regulating the applied electric current and holding electrodes at constant 233 potential (GCD-CPM). This method was employed to delve into the charging-discharging 
234 dynamics of the open electrode and nanoporous electrode systems, compared with traditional

235 GCD-CCM simulations. For open electrode systems, the GCD curve and dynamic EDL 236 structure by these two methods agree well. For nanoporous electrode systems, GCD-CCM 237 produces unphysical phenomena, including parabolic-like potential distributions on 238 electrodes, excessive ion diffusivity, and overmuch heat generation. In contrast, GCD-CPM well captures the charging dynamics, confirmed by experimental GCD tests with a bridge of

240 the dimensionless transmission line model. The modeling with GCD-CPM discloses the 241 hysteresis of ion adsorption-desorption during charging and discharging, which is found to 242 originate from the electrolyte response lagging behind the electrode charge response to the 243 polarization. In this work, the flexibility of the electrode is not considered in our model, 244 which could work for electrodes with neglecting deformation during charging and 245 discharging, while for electrodes having non-negligible expansion such as MXenes ${ }^{47,}{ }^{48}$, 246 electrode flexibility needs to be taken into account.

247 The GCD-CPM developed here could not only model the charging and discharging 248 process of supercapacitors with applying electric currents of any form, but also can be 249 combined with previous CPM methods in the potentiostatic mode to investigate complex 250 charging modes at the molecular level, thereby helping to gain deep insights into dynamics 251 mechanisms and optimal charging modes. It may also be used for other applications 252 involving the dynamic formation and release of double layers, such as capacitive 253 deionization $^{49}$, $^{\text {battery }}{ }^{50}$, superlubricity $^{51}$, and electrolyte gating ${ }^{52}$.

\section{Methods}

\section{GCD-CPM for modeling galvanostatic charge-discharge}

256 We consider a classical electrochemical system containing $N$ immobile electrode atoms 257 (including $N_{\text {pos }}$ positive electrode atoms and $N_{\text {neg }}$ negative electrode atoms) and $M$ 258 mobile electrolyte atoms. The constant potential method considers Gaussian charge 259 distributions for electrode atoms and point charges for electrolyte atoms as usual ${ }^{8,9}$. The 260 fluctuating charges on electrodes with a magnitude $\mathbf{q}$ are extra degrees of freedom to keep 
261 electrodes equipotential. The electrostatic energy of the system $U_{\text {ele }}$ is a function of atom positions $\mathbf{X}$ and electrode charge magnitude $\mathbf{q}$ written as $9,16,53$

$$
U_{e l e}(\mathbf{X}, \mathbf{q})=\frac{\mathbf{q}^{\mathbf{T}} \mathbf{A q}}{2}-\mathbf{q}^{\mathbf{T}} \mathbf{B}(\mathbf{X})+C(\mathbf{X}) .
$$

264 The first and second terms in the right-hand side of Eq. 2 represent the electrode-electrode 265 and electrode-electrolyte interactions, respectively, and the last term describes the electrolyte-electrolyte interaction. Matrix A depends on the position of electrode atoms, and its size is $N \times N$; $\mathbf{B}$ is a vector of $N \times 1 ; C$ is a scalar. Details of $\mathbf{A}, \mathbf{B}$, and $C$ can be found in the literature ${ }^{9,16,53}$. The potential on each electrode atom is $\frac{\partial U_{\text {ele }}}{\partial q_{i}}(i$ for the electrode atom index). To model the galvanostatic charge-discharge, the total electrode charge is constrained as $Q(t)=\sum_{i=1}^{N_{\text {pos }}} q_{i}$ and $\sum_{i=N_{\text {pos }}+1}^{N_{\text {pos }}+N_{\text {neg }}} q_{i}=-Q(t)$. In this work, $Q(t)$ is a triangular wave for GCD due to the applied square-wave current (Fig. 1d). To keep electrode equipotential at any time step and meet the constraints, we need to minimize the energy

$$
U_{t}(\mathbf{X}, \mathbf{q})=U_{e l e}-\mathbf{q}^{\mathrm{T}} \boldsymbol{\phi}+Q(t) \phi_{p o s}-Q(t) \phi_{n e g} .
$$

274 The vector $\boldsymbol{\phi}^{\mathbf{T}}=\left[\phi_{\text {pos }} \mathbf{I}_{\text {pos }}, \phi_{\text {neg }} \mathbf{I}_{\text {neg }}\right]$ is for the potentials on all electrode atoms, where $275 \phi_{\text {pos }}\left(\phi_{n e g}\right)$ is the potential on the positive (negative) electrode. $\mathbf{I}_{\text {pos }}\left(\mathbf{I}_{\text {neg }}\right)$ is a vector of 276 size $1 \times N_{\text {pos }}\left(1 \times N_{\text {neg }}\right)$, whose elements are all one. Similar to the prior CPM simulations 277 with step-like potentials, we solve a set of linear equations to minimize $U_{t}$ at each time step, then obtain $\phi_{\text {pos }}, \phi_{\text {neg }}$, and electrode atom charges q. Mathematically, we have

$$
\mathbf{M x}=\mathbf{n} .
$$

280 The size of matrix $\mathbf{M}=\left[\begin{array}{ll}\mathbf{A} & \mathbf{E} \\ \mathbf{D} & \mathbf{0}\end{array}\right]$ is $(N+2) \times(N+2)$ where $\quad \mathbf{D}=\left[\begin{array}{cc}\mathbf{I}_{\mathbf{p o s}} & \mathbf{0} \\ \mathbf{0} & \mathbf{I}_{\mathbf{n e g}}\end{array}\right]$, $281 \mathbf{E}=\left[\begin{array}{cc}-\mathbf{I}_{\text {pos }}^{\mathbf{T}} & \mathbf{0} \\ \mathbf{0} & -\mathbf{I}_{\text {neg }}^{\mathbf{T}}\end{array}\right]$. Unknowns of the linear equations are $\mathbf{x}^{\mathbf{T}}=\left[\mathbf{q}^{\mathbf{T}}, \phi_{\text {pos }}, \phi_{\text {neg }}\right]$, and $282 \mathbf{n}^{\mathbf{T}}=\left[-\mathbf{B}^{\mathbf{T}}, Q(t),-Q(t)\right]$. Matrices $\mathbf{A}$ and $\mathbf{B}$ are the same as the prior CPM simulations 283 with step-like potentials ${ }^{9,16,53}$. 
285 For the supercapacitor with open electrodes, each electrode is modeled by three layers of 286 graphene sheets (Fig. 1a). The simulation box has a volume of $4.254 \times 4.176 \times 48 \mathrm{~nm}^{3}$, with a 287 distance of $10 \mathrm{~nm}$ between the innermost layers of the positive and negative electrodes. For 288 the supercapacitor with nanoporous electrodes, each electrode is modeled as a slit-shaped pore whose walls are made by three layers of graphene sheets (Fig. 1b). The pore size of 0.67 $\mathrm{nm}$ is defined as the accessible gap between the two innermost slit sheets in contact with the electrolyte, and the pore length is $8 \mathrm{~nm}$. The dimensions of the simulation box are $4.1 \mathrm{~nm}, 3$ $\mathrm{nm}$, and $32 \mathrm{~nm}$. The simulation box of all systems is sufficiently large to ensure that the central region of the electrolyte reservoir is in a bulk-like state. The Lennard-Jones model of carbon atom $^{54}$ is taken for the electrode, and a coarse-grained model (Fig. 1b), which provides accurate thermodynamic and dynamic properties, is adopted for ionic liquid $[\mathrm{EMIM}]\left[\mathrm{BF}_{4}\right]^{55}$.

All simulations are performed in the NVT ensemble using a customized MD software $\operatorname{GROMACS}^{56}$ (see 'Code availability' for more information), with a time step of 2 fs and a Nosé-Hoover thermostat ${ }^{57}$ at a temperature of $400 \mathrm{~K}$. The heat generation during the

300 galvanostatic charge-discharge is calculated as the energy removed from the system to maintain its temperature ${ }^{57,58}$. To avoid energy drift and attain accurate heat generation, our simulations are in double-precision ${ }^{56}$. A cutoff length of $1.2 \mathrm{~nm}$ is chosen to calculate non-electrostatic interactions and electrostatic interactions in real space. The electrostatic interactions in reciprocal space are computed using the particle mesh Ewald method ${ }^{59}$ with a fast Fourier transform grid spacing of $0.1 \mathrm{~nm}$. For open electrode systems, the slab correction is adopted in electrostatic interactions ${ }^{5}$.

307 For each simulation, the system is first run for $40 \mathrm{~ns}$ to reach equilibrium at the potential 308 of zero surface charge. Then a square-wave current with 30 cycles is applied on 309 supercapacitors (Fig. 1b). We averaged the results of the last ten cycles to obtain results for a 310 stable cycle, so that the time mentioned above refers to the time relative to the beginning of 311 the averaged cycle. For the open electrode system, the charge density of the positive electrode 
312 grows linearly from 0 to $9 \mu \mathrm{C} / \mathrm{cm}^{2}$ in the first half of each cycle and then decreases linearly to

3130 in the second half cycle. The period of applied electric current is set as 50, 100, and 500 ps.

314 For the nanoporous electrode system, the surface charge density on the electrode is set to

315 change linearly between $0 \sim 4.96 \mu \mathrm{C} / \mathrm{cm}^{2}$, with the periods of 200,1000 , and 3000 ps. For

316 GCD-CPM, the charge on electrode atoms is obtained using the method mentioned above; for

317 GCD-CCM, the potential at each electrode atom is computed as the sum of the potentials

318 from real space and reciprocal space. To ensure the accuracy of the simulation results, five

319 independent simulations are run with different initial configurations for each system.

320 Transmission line model for galvanostatic charge-discharge

321 The transmission line model (TLM) describes the potential at time $t$ and location along pore

322 axis $z$, expressed in the equation as follows 39,40

$$
\frac{\partial \phi}{\partial t}=\frac{l^{2}}{R C} \frac{\partial^{2} \phi}{\partial z^{2}}
$$

324

325

where $S$ is a square-wave function whose period and amplitude are both one. Equation 6 a indicates the pore is uncharged at $t=0$. The boundary condition in Eq. $6 \mathrm{~b}$ is the zero normal

332 flux at $z=l$ (corresponds to the middle position of the open pore in our simulations), and

333 the boundary in Eq. 6c indicates the applied square-wave current with a period $P$ and an

334 amplitude $I_{0}$, as shown in Fig. 1d. We define the dimensionless potential $\bar{\Phi}=\phi / I_{0} R$, 335 where $\phi$ is potential, $l$ is pore length, and $R$ and $C$ are the resistance and capacitance of the pore, respectively. For the galvanostatic charge-discharge, the initial and boundary conditions are

$$
\left.\frac{\partial \phi}{\partial z}\right|_{z=0}=-\frac{I_{0} R}{l} S\left(\frac{t}{P}\right)
$$


336 constant), and the dimensionless period $\alpha=P / \tau$. Hence, Eq. 5 can be written in

337 dimensionless form as

$$
\frac{\partial \bar{\Phi}}{\partial \overline{\mathrm{T}}}=\frac{\partial^{2} \bar{\Phi}}{\partial Z^{2}}
$$

339 The initial and boundary conditions become as

$$
\begin{gathered}
\left.\bar{\Phi}\right|_{\overline{\mathrm{T}}=0}=0, \\
\left.\frac{\partial \Phi}{\partial Z}\right|_{Z=1}=0, \\
\left.\frac{\partial \Phi}{\partial Z}\right|_{Z=0}=-S\left(\frac{\overline{\mathrm{T}}}{\alpha}\right) .
\end{gathered}
$$

343 Accordingly, the potential difference between the positive and negative electrodes $\Delta \bar{\Phi}(\overline{\mathrm{T}})$

344 changing with time during the charging-discharging process could be obtained, which is the

345 GCD curve and given by

$$
\Delta \bar{\Phi}(\overline{\mathrm{T}})=\frac{2}{3}+2 \overline{\mathrm{T}}-\sum_{n=1}^{\infty} \frac{4}{n^{2} \pi^{2}} e^{-n^{2} \pi^{2} \bar{T}}+4 \sum_{m=1}^{\infty}(-1)^{m} \Phi_{\text {step }}(m, \overline{\mathrm{T}})
$$

347 where

$$
\Phi_{\text {step }}(m, \overline{\mathrm{T}})=\left[\frac{1}{3}+\left(\overline{\mathrm{T}}-\frac{m \alpha}{2}\right)\right] \theta\left(\overline{\mathrm{T}}-\frac{m \alpha}{2}\right)-\sum_{n=1}^{\infty} \frac{2}{n^{2} \pi^{2}} e^{-\left(\overline{\mathrm{T}}-\frac{m \alpha}{2}\right) n^{2} \pi^{2}} \theta\left(\overline{\mathrm{T}}-\frac{m \alpha}{2}\right),
$$

and $\theta$ is the Heaviside step function. Therefore, the relation between $\Delta \bar{\Phi}$ and $\overline{\mathrm{T}}$, described by Eq. 9, reveals that the dimensionless GCD curve is only determined by the dimensionless period $\alpha$ (i.e., the ratio of the period to the charging time constant of the system).

\section{Data availability}

354 The modeling results of galvanostatic charge-discharge of supercapacitors using CPM and CCM approaches and electrochemical measurements are available at ...

\section{Code availability}

357 The code modeling galvanostatic charge-discharge process in molecular dynamics simulation using CPM and CCM approaches are available from ... 
1. Wang X., Salari M., Jiang D., Chapman Varela J., Anasori B., Wesolowski D. J., Dai S., Grinstaff M. W., Gogotsi Y. Electrode material-ionic liquid coupling for electrochemical energy storage. Nature Reviews Materials 2020, 5(11): 787-808.

2. Simon P., Gogotsi Y. Perspectives for electrochemical capacitors and related devices. Nature Materials 2020, 19(11): 1151-1163.

3. Salanne M., Rotenberg B., Naoi K., Kaneko K., Taberna P. L., Grey C. P., Dunn B., Simon 1(6): 16070.

4. Bedrov D., Piquemal J.-P., Borodin O., MacKerell A. D., Roux B., Schröder C. Molecular dynamics simulations of ionic liquids and electrolytes using polarizable force fields. Chemical Reviews 2019, 119(13): 7940-7995.

5. Scalfi L., Salanne M., Rotenberg B. Molecular simulation of electrode-solution interfaces. Annual Review of Physical Chemistry 2021, 72(1): 189-212.

6. Joly L., Ybert C., Trizac E., Bocquet L. Hydrodynamics within the electric double layer on slipping surfaces. Physical Review Letters 2004, 93(25): 257805.

7. Yang L., Fishbine B. H., Migliori A., Pratt L. R. Molecular simulation of electric double-layer capacitors based on carbon nanotube forests. Journal of the American Chemical Society 2009, 131(34): 12373-12376.

8. Siepmann J. I., Sprik M. Influence of surface topology and electrostatic potential on water/electrode systems. The Journal of Chemical Physics 1995, 102(1): 511-524.

9. Reed S. K., Lanning O. J., Madden P. A. Electrochemical interface between an ionic liquid and a model metallic electrode. Journal of Chemical Physics 2007, 126(8): 084704.

10. Vatamanu J., Borodin O., Smith G. D. Molecular insights into the potential and temperature dependences of the differential capacitance of a room-temperature ionic liquid at graphite electrodes. Journal of the American Chemical Society 2010, 132(42): 14825-14833.

11. Merlet C., Rotenberg B., Madden P. A., Taberna P. L., Simon P., Gogotsi Y., Salanne M. On the molecular origin of supercapacitance in nanoporous carbon electrodes. Nature Materials 2012, 11(4): 306-310.

12. Kondrat S., Wu P., Qiao R., Kornyshev A. A. Accelerating charging dynamics in subnanometre pores. Nature Materials 2014, 13(4): 387-393.

13. Bi S., Banda H., Chen M., Niu L., Chen M., Wu T., Wang J., Wang R., Feng J., Chen T., Dincă M., Kornyshev A. A., Feng G. Molecular understanding of charge storage and charging dynamics in supercapacitors with MOF electrodes and ionic liquid electrolytes. Nature Materials 2020, 19(5): 552-558.

14. Dufils T., Jeanmairet G., Rotenberg B., Sprik M., Salanne M. Simulating electrochemical systems by combining the finite field method with a constant potential electrode. Physical Review Letters 2019, 123(19): 195501.

15. Chen M., Wu J., Ye T., Ye J., Zhao C., Bi S., Yan J., Mao B., Feng G. Adding salt to expand voltage window of humid ionic liquids. Nature Communications 2020, 11(1): 5809. 
16. Wang Z., Yang Y., Olmsted D. L., Asta M., Laird B. B. Evaluation of the constant potential method in simulating electric double-layer capacitors. Journal of Chemical Physics 2014, 141(18): 184102.

17. Feng G., Jiang D., Cummings P. T. Curvature effect on the capacitance of electric double layers at ionic liquid/onion-like carbon interfaces. Journal of Chemical Theory and Computation 2012, 8(3): 1058-1063.

18. Yang J. Y., Bo Z., Yang H. C., Qi H. L., Kong J., Yan J. H., Cen K. F. Reliability of constant charge method for molecular dynamics simulations on EDLCs in nanometer and sub-nanometer spaces. Chemelectrochem 2017, 4(10): 2486-2493.

19. Merlet C., Pean C., Rotenberg B., Madden P. A., Simon P., Salanne M. Simulating supercapacitors: Can we model electrodes as constant charge surfaces? Journal of Physical Chemistry Letters 2013, 4(2): 264-268.

20. Pean C., Merlet C., Rotenberg B., Madden P. A., Taberna P. L., Daffos B., Salanne M., Simon P. On the dynamics of charging in nanoporous carbon-based supercapacitors. ACS Nano 2014, 8(2): 1576-1583.

21. He Y. D., Huang J. S., Sumpter B. G., Kornyshev A. A., Qiao R. Dynamic charge storage in ionic liquids-filled nanopores: Insight from a computational cyclic voltammetry study. Journal of Physical Chemistry Letters 2015, 6(1): 22-30.

22. Breitsprecher K., Holm C., Kondrat S. Charge me slowly, I am in a hurry: Optimizing charge-discharge cycles in nanoporous supercapacitors. ACS Nano 2018, 12(10): 9733-9741.

23. Breitsprecher K., Janssen M., Srimuk P., Mehdi B. L., Presser V., Holm C., Kondrat S. How to speed up ion transport in nanopores. Nature Communications 2020, 11(1): 6085.

24. Li H., Zhang X., Peng J., He J., Huang Z., Wang J. Cooperative CC-CV charging of supercapacitors using multicharger systems. IEEE Transactions on Industrial Electronics 2020, 67(12): 10497-10508.

25. AbdelAty A. M., Fouda M. E., Elbarawy M. T. M. M., Radwan A. G. Optimal charging and discharging of supercapacitors. Journal of the Electrochemical Society 2020, 167(11): 110521 .

26. Noori A., El-Kady M. F., Rahmanifar M. S., Kaner R. B., Mousavi M. F. Towards establishing standard performance metrics for batteries, supercapacitors and beyond. Chemical Society Reviews 2019, 48(5): 1272-1341.

27. Zhang S., Pan N. Supercapacitors performance evaluation. Advanced Energy Materials 2015, 5(6): 1401401.

28. Stoller M. D., Ruoff R. S. Best practice methods for determining an electrode material's performance for ultracapacitors. Energy \& Environmental Science 2010, 3(9): 1294-1301.

29. Dufils T., Sprik M., Salanne M. Computational amperometry of nanoscale capacitors in molecular simulations. 2021, arXiv:2104.03177.

30. Jiang X., Huang J., Zhao H., Sumpter B. G., Qiao R. Dynamics of electrical double layer formation in room-temperature ionic liquids under constant-current charging conditions. Journal of Physics: Condensed Matter 2014, 26(28): 284109. 

$\mathrm{P}$. Effects of functional groups and anion size on the charging mechanisms in layered electrode materials. Energy Storage Materials 2020, 33: 460-469.

32. Limmer D. T., Merlet C., Salanne M., Chandler D., Madden P. A., van Roij R., Rotenberg B. Charge fluctuations in nanoscale capacitors. Physical Review Letters 2013, 111(10): 106102.

33. Bazant M. Z., Storey B. D., Kornyshev A. A. Double layer in ionic liquids: Overscreening versus crowding. Physical Review Letters 2011, 106(4): 046102.

34. Fedorov M. V., Kornyshev A. A. Ionic liquids at electrified interfaces. Chemical Reviews 2014, 114(5): 2978-3036.

35. Dong K., Liu X., Dong H., Zhang X., Zhang S. Multiscale studies on ionic liquids. Chemical Reviews 2017, 117(10): 6636-6695.

36. Kondrat S., Kornyshev A. Charging dynamics and optimization of nanoporous supercapacitors. The Journal of Physical Chemistry C 2013, 117(24): 12399-12406.

37. Burke A., Miller M. Testing of electrochemical capacitors: Capacitance, resistance, energy density, and power capability. Electrochimica Acta 2010, 55(25): 7538-7548.

38. González A., Goikolea E., Barrena J. A., Mysyk R. Review on supercapacitors: Technologies and materials. Renewable and Sustainable Energy Reviews 2016, 58: 1189-1206.

39. De Levie R. On porous electrodes in electrolyte solutions: I. Capacitance effects. Electrochimica Acta 1963, 8(10): 751-780.

40. Janssen M. Transmission line circuit and equation for an electrolyte-filled pore of finite length. Physical Review Letters 2021, 126(13): 136002.

41. Gupta A., Zuk P. J., Stone H. A. Charging dynamics of overlapping double layers in a cylindrical nanopore. Physical Review Letters 2020, 125(7): 076001.

42. Mirzadeh M., Gibou F., Squires T. M. Enhanced charging kinetics of porous electrodes: Surface conduction as a short-circuit mechanism. Physical Review Letters 2014, 113(9): 097701.

43. Tsai W. Y., Taberna P. L., Simon P. Electrochemical quartz crystal microbalance (EQCM) study of ion dynamics in nanoporous carbons. Journal of the American Chemical Society 2014, 136(24): 8722-8728.

44. Levi M. D., Salitra G., Levy N., Aurbach D., Maier J. Application of a quartz-crystal microbalance to measure ionic fluxes in microporous carbons for energy storage. Nature Materials 2009, 8(11): 872-875.

45. Platek-Mielczarek A., Frackowiak E., Fic K. Specific carbon/iodide interactions in electrochemical capacitors monitored by EQCM technique. Energy \& Environmental Science 2021, 14(4): 2381-2393.

46. Weidlich C., Mangold K. M., Jüttner K. EQCM study of the ion exchange behaviour of polypyrrole with different counterions in different electrolytes. Electrochimica Acta 2005, 50(7): 1547-1552.

47. Wang X., Mathis T. S., Li K., Lin Z., Vlcek L., Torita T., Osti N. C., Hatter C., Urbankowski P., Sarycheva A., Tyagi M., Mamontov E., Simon P., Gogotsi Y. Influences 

241-248.

48. Lukatskaya M. R., Mashtalir O., Ren C. E., Dall'Agnese Y., Rozier P., Taberna P. L., Naguib M., Simon P., Barsoum M. W., Gogotsi Y. Cation intercalation and high volumetric capacitance of two-dimensional titanium carbide. Science 2013, 341(6153): 1502-1505.

49. Srimuk P., Su X., Yoon J., Aurbach D., Presser V. Charge-transfer materials for electrochemical water desalination, ion separation and the recovery of elements. Nature Reviews Materials 2020, 5(7): 517-538.

50. Swift M. W., Swift J. W., Qi Y. Modeling the electrical double layer at solid-state electrochemical interfaces. Nature Computational Science 2021, 1(3): 212-220.

51. Hod O., Meyer E., Zheng Q., Urbakh M. Structural superlubricity and ultralow friction across the length scales. Nature 2018, 563(7732): 485-492.

52. Ye J. T., Inoue S., Kobayashi K., Kasahara Y., Yuan H. T., Shimotani H., Iwasa Y. Liquid-gated interface superconductivity on an atomically flat film. Nature Materials 2010, 9(2): 125-128.

53. Gingrich T. R., Wilson M. On the Ewald summation of gaussian charges for the simulation of metallic surfaces. Chemical Physics Letters 2010, 500(1-3): 178-183.

54. Cornell W. D., Cieplak P., Bayly C. I., Gould I. R., Merz K. M., Ferguson D. M., Spellmeyer D. C., Fox T., Caldwell J. W., Kollman P. A. A second generation force field for the simulation of proteins, nucleic acids, and organic molecules. Journal of the American Chemical Society 1995, 117(19): 5179-5197.

55. Merlet C., Salanne M., Rotenberg B. New coarse-grained models of imidazolium ionic liquids for bulk and interfacial molecular simulations. The Journal of Physical Chemistry C 2012, 116(14): 7687-7693.

56. Hess B., Kutzner C., van der Spoel D., Lindahl E. Gromacs 4: Algorithms for highly efficient, load-balanced, and scalable molecular simulation. Journal of Chemical Theory and Computation 2008, 4(3): 435-447.

57. Evans D. J., Holian B. L. The Nose-Hoover thermostat. The Journal of Chemical Physics 1985, 83(8): 4069-4074.

58. Esposito M., Monnai T. Nonequilibrium thermodynamics and Nose-Hoover dynamics. The Journal of Physical Chemistry B 2011, 115(18): 5144-5147.

59. Essmann U., Perera L., Berkowitz M. L., Darden T., Lee H., Pedersen L. G. A smooth particle mesh Ewald method. The Journal of Chemical Physics 1995, 103(19): 8577-8593. 


\section{Acknowledgments}

523 Authors at HUST acknowledge the funding support from the National Natural Science

524 Foundation of China $(51876072,51836003)$ and the Hubei Provincial Natural Science

525 Foundation of China (2020CFA093). The work at HUST is also supported by the Program for

526 HUST Academic Frontier Youth Team.

\section{Author contributions}

528 G.F. conceived this research and designed the simulation and experiment. L.Z. performed all 529 simulations; T.Z.W. carried out the experiment; L.Z. and T.Y. derived dimensionless

530 transmission line model. L.Z. and T.Z.W. drafted the manuscript. All authors contributed to

531 the discussion of results, editing, and revising the paper.

532 Ethics declarations

533 Competing interests

534 The authors declare no competing interests.

535 Additional information

536 Supplementary Information is available for this paper at ... 


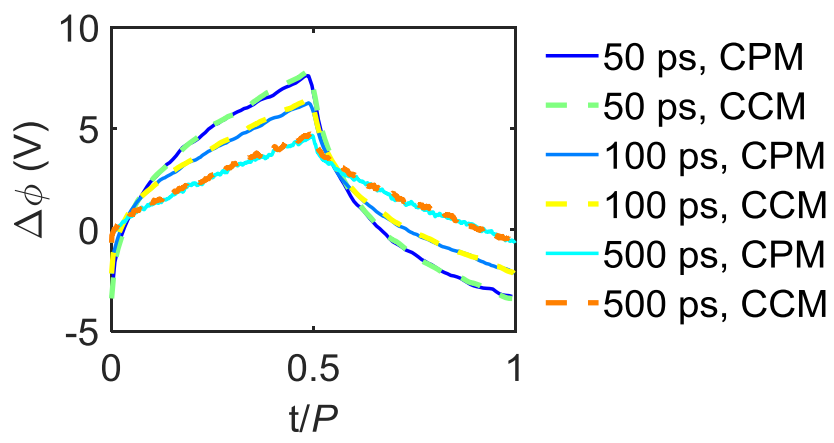

539 Extended Data Fig. 1 | GCD curves with various periods for open electrode systems. 
a
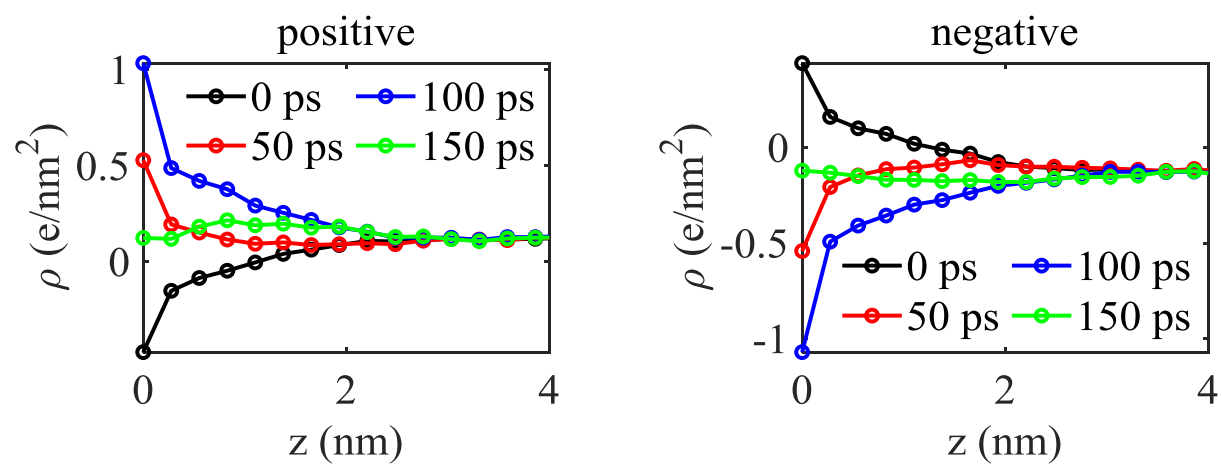

b
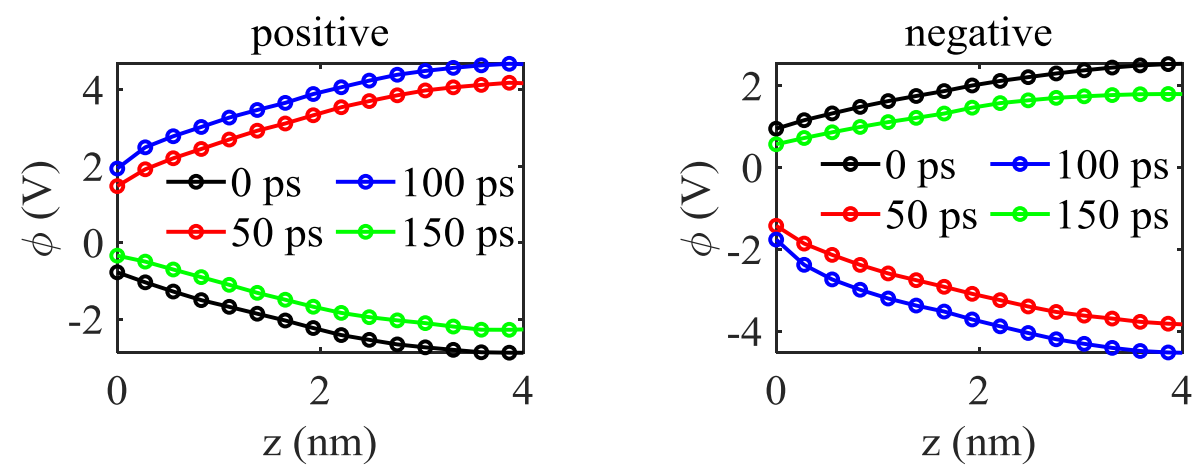

Extended Data Fig. 2 | Distribution of charge and potential on electrodes atoms in nanoporous

542 electrode systems. a, Spatial distribution of electrode atom charge along pore axis for GCD-CPM

543 simulations. b, Spatial distribution of electrode atom potential along pore axis for GCD-CCM

544 simulations. The data is not at one moment, but a statistic average, which each time counts the data of 5455 ps around it. 


\section{Supplementary Files}

This is a list of supplementary files associated with this preprint. Click to download.

- GCDSIV6.6.pdf

- flatFengepc.pdf 